PROCEEDINGS OF THE

AMERICAN MATHEMATICAL SOCIETY

Volume 131, Number 4, Pages 1257-1266

S 0002-9939(02)06688-1

Article electronically published on October 1, 2002

\title{
BV ESTIMATES OF LAX-FRIEDRICHS' SCHEME FOR A CLASS OF NONLINEAR HYPERBOLIC CONSERVATION LAWS
}

\author{
TONG YANG, HUIJIANG ZHAO, AND CHANGJIANG ZHU
}

(Communicated by Suncica Canic)

\begin{abstract}
We give uniform BV estimates and $L^{1}$-stability of Lax-Friedrichs' scheme for a class of $n \times n$ systems of strictly hyperbolic conservation laws whose integral curves of the eigenvector fields are straight lines, i.e., Temple class, under the assumption of small total variation. This implies that the approximate solutions generated via the Lax-Friedrichs' scheme converge to the solution given by the method of vanishing viscosity or the Godunov scheme, and then the Glimm scheme or the wave front tracking method.
\end{abstract}

\section{INTRODUCTION}

Consider the one dimensional Cauchy problem for a system of nonlinear hyperbolic conservation laws

$$
u_{t}+f(u)_{x}=0, \quad x \in \mathbf{R}, \quad t>0
$$

with initial data

$$
u(x, 0)=u_{0}(x), \quad x \in \mathbf{R} .
$$

Here $f(u)$ is a smooth map from a domain $\Omega \subset \mathbf{R}^{n}$ into $\mathbf{R}^{n}$.

The study of the Cauchy problem (1.1), (1.2), especially the well-posedness theory of the entropy solutions, has been one of the most important problems. This problem for a scalar conservation law was solved perfectly in the 1960's [3], [9], [12], [13] mainly because of the maximum principle. Progress on systems of conservation laws was made only recently. First, A. Bressan [3], 6] and his group, T.-P. Liu and T. Yang [6], 11] obtained the $L^{1}$-stability of the entropy solution to

Received by the editors December 5, 2001.

1991 Mathematics Subject Classification. Primary 35L60, 35L65, 65N12.

Key words and phrases. Hyperbolic systems of conservation laws, Lax-Friedrichs' scheme, BV estimates.

The first author was supported in part by the Research Grants Council of the Hong Kong Special Administrative Region, China (CityU 1032/98P).

The second author was supported in part by a grant from the National Natural Science Foundation of China (The Youth Foundation) and a grant from the Chinese Academy of Sciences entitled "Yin Jin Guo Wai Jie Chu Ren Cai Ji Jing".

The third author was supported in part by a grant from the National Natural Science Foundation of China \#10171037 and two grants from the Ministry of Education of China entitled "Liu Xue Hui Guo Ren Yuan Ji Jing" and "Gao Deng Xue Xiao Zhong Dian Shi Yang Shi Ji Jing", respectively. 
systems of hyperbolic conservation laws obtained via the Glimm scheme [8] or wave front tracking method [3] when each characteristic field is either genuinely nonlinear or linearly degenerate. Second, S. Bianchini and A. Bressan [1] established the uniform BV estimates to general strictly hyperbolic systems with artificial viscosity and consequently showed that the solution obtained via the vanishing viscosity method coincides with those obtained via the Glimm scheme or wave front tracking method.

It is well-known that most of the numerical schemes contain numerical viscosities. When the grid size tends to zero, the numerical viscosity tends to zero. This is a discrete version of the vanishing viscosity problem which is more difficult than the continuous version because the general existence result on discrete travelling wave solutions is not known. Also, the decomposition of the solutions at each point into travelling wave solutions is the main new idea in the recent approach in [1] for the continuous version of vanishing viscosity. Therefore, the well-posedness theory for numerical scheme is largely open. As a recent result in this direction, A. Bressan and H. K. Jenssen [4] gave the uniform BV estimates on the approximate solutions generated by the Godunov scheme for a class of systems of hyperbolic conservation laws whose integral curves of the eigenvector fields are straight lines, i.e., Temple class. The main result in this paper is to give uniform BV estimates on Lax-Friedrichs' scheme for the same class of systems as in [4].

Based on the theory of compensated compactness [13, R. J. DiPerna 7] proved the convergence of Lax-Friedrichs' scheme for $2 \times 2$ systems of strictly hyperbolic, genuinely nonlinear conservation laws provided that the a priori $L^{\infty}$ estimate on the approximate solutions can be obtained. However, the regularity of these solutions is not enough to obtain the well-posedness of the solutions. On the other hand, a uniform BV estimate of the approximate solutions generated by Lax-Friedrichs' scheme is sufficient to deduce the desired well-posedness result. In the previous work, the BV estimates on the approximate solutions constructed by Lax-Friedrichs' scheme was only proved for scalar equations [9, [12. To our knowledge, even under the assumption of small total variation of the initial data, the corresponding result for the system is not known.

As a first step toward the understanding of the uniform BV estimates of LaxFriedrichs' scheme for nonlinear systems of hyperbolic conservation laws, we show in this paper that, under the same straight line assumption on wave curves as in 2 . and [4], the approximate solutions constructed by Lax-Friedrichs' scheme converge to the same solution as obtained by vanishing viscosity [2] or by the Godunov scheme [4], and then the Glimm scheme [8] or the wave front tracking method [3].

To state our main result precisely, we first list the assumptions as follows. Let $D f(u)$, the Jacobian matrix of $f(u)$, have $n$ real and distinct eigenvalues $\lambda^{k}(u), k=$ $1,2, \cdots, n, u \in \Omega$. By possibly restricting to a smaller domain $\Omega$ and by performing the linear change of independent coordinates

$$
t^{\prime}=2 \bar{\lambda} t, \quad x^{\prime}=x+\bar{\lambda} t
$$

where $\bar{\lambda}=\max _{u \in \Omega, 1 \leq i \leq n}\left|\lambda^{k}(u)\right|$, we can assume that there are constants $\bar{\lambda}^{0}=0<$ $\bar{\lambda}^{1}<\cdots<\bar{\lambda}^{n-1}<\overline{\bar{\lambda}}^{n}=1$ such that

$$
\bar{\lambda}^{i-1}<\lambda^{i}(u)<\bar{\lambda}^{i}, \quad u \in \Omega, \quad i=1,2, \cdots, n .
$$


The corresponding right and left eigenvectors are denoted by $r^{k}(u), l^{k}(u)$, respectively, and normalized so that $\left|r^{k}(u)\right| \equiv 1, r^{i}(u) \cdot l^{j}(u)=\delta_{i j}$. Here $\delta_{i j}$ is the Kronecker symbol.

The basic assumption throughout the paper is that the integral curves of the eigenvector fields are straight lines, i.e.,

$$
\left(D r^{k}(u)\right) \cdot r^{k}(u)=0, u \in \Omega, k=1,2, \cdots, n,
$$

where $D r^{k}(u)$ denotes the Jacobian matrix of $r^{k}(u)$. (1.4) implies that the shock curves and the rarefaction curves coincide and are straight lines in $\Omega$; see [14].

By assumption (1.3), the Courant-Friedrichs-Lewy condition is satisfied with equal time and space step $\Delta x=\Delta t$. Thus, without loss of generality, we assume $\Delta x=\Delta t$ in the following discussion for simplicity. Denote the value of the approximate solution in the $i-t h$ cell $\left[\left(i-\frac{1}{2}\right) \Delta x,\left(i+\frac{1}{2}\right) \Delta x\right)$ at time $j \Delta t$ by $u_{i}^{j}$. Lax-Friedrichs' scheme is given inductively as follows (cf. [9]): At time $t=0$, let $u_{i}^{0}$ denote the average over the $i-t h$ cell of the initial data $u_{0}(x)$,

$$
u_{i}^{0}=\frac{1}{\Delta x} \int_{\left(i-\frac{1}{2}\right) \Delta x}^{\left(i+\frac{1}{2}\right) \Delta x} u_{0}(\xi) d \xi .
$$

Given the values $u_{i-1}^{j}$ and $u_{i+1}^{j}$ in the $(i-1)-t h$ and $(i+1)-t h$ cells, at time $j \Delta t, j \geq 0, u_{i}^{j+1}$ in the $i-t h$ cell at time $(j+1) \Delta t$ is given by

$$
u_{i}^{j+1}=\frac{1}{2}\left(u_{i+1}^{j}+u_{i-1}^{j}\right)-\frac{1}{2}\left(f\left(u_{i+1}^{j}\right)-f\left(u_{i-1}^{j}\right)\right) .
$$

It is easy to see that for each $\Delta x$, the scheme gives an approximate solution $u_{\Delta x}$ of (1.1) and (1.2) for all $(x, t) \in \mathbf{R} \times \mathbf{R}^{+}$provided that the values $u_{i}^{j}$ remain in $\Omega$.

For each fixed time step $j \Delta t$, we use $u(j)$ to denote the discrete function: $i \rightarrow$ $u_{i}^{j}, i \in \mathbf{Z}$. The total variation of $u(j)$ is then given by

$$
\mathrm{T} . \mathrm{V} \cdot[u(j)]=\sum_{i \in \mathbf{Z}}\left|u_{i}^{j}-u_{i-1}^{j}\right| \cdot
$$

Based on the above notations, our main result can be stated in the following.

Theorem 1.1. Suppose that (1.1) is strictly hyperbolic for each $u \in \Omega$ and the normalized eigenvectors satisfy (1.4). Then there exist constants $\delta_{0}>0, \delta_{1}>0$ such that the following holds. For each initial data $u_{0}(x)$ with

$$
\text { T.V. }\left[u_{0}(x)\right]<\delta_{0},
$$

the corresponding solution $u_{i}^{j}$ of Lax-Friedrichs' scheme (1.5) is well defined for all time steps $j \in \mathbf{Z}^{+}$and satisfies

$$
\text { T.V. }[u(j)]<\delta_{1}, \text { for all } j \in \mathbf{Z}^{+} \text {. }
$$

Furthermore, there exists a constant $L$ such that for all pairs of initial data $u_{0}(x)$, $v_{0}(x)$ satisfying (1.6), the corresponding solutions $u(j)$ and $v\left(j^{\prime}\right)$ satisfy

$$
\sum_{i=-\infty}^{+\infty}\left|u_{i}^{j}-v_{i}^{j^{\prime}}\right| \Delta x \leq L \cdot\left(\left|j-j^{\prime}\right| \cdot \Delta t+\left\|u_{0}(x)-v_{0}(x)\right\|_{L^{1}}\right) .
$$

As a direct consequence of Theorem 1.1, we can deduce that the approximate solutions constructed via Lax-Friedrichs' scheme converge to the same solution as given by the method of vanishing viscosity [2] or the Godunov scheme [4], and then the Glimm scheme [8] or the wave front tracking method [3]. 
Before concluding this section, we give the main idea in proving Theorem 1.1. Due to the straight line assumption (1.4), the increase in total variation induced by Lax-Friedrichs' scheme comes only from the terms involving two different characteristic families and consequently the techniques developed in [4] can be used to control the creation of new oscillations in the approximate solutions generated by the scheme. It is worth pointing out that the straight line assumption (1.4) plays an essential role in our analysis and new tools are needed to study the influence of those terms from the interaction of the same family in the general case.

\section{BV estimates}

Let us first give some basic properties of the Riemann problem for (1.1) under the assumption (1.4), i.e., (1.1) with initial data

$$
u_{0}(x)= \begin{cases}u_{-}, & \text {for } x<0, \\ u_{+}, & \text {for } x>0 .\end{cases}
$$

Given $u_{-}, u_{+} \in \Omega$, by strict hyperbolicity, the assumption (1.4), and the Implicit Function Theorem there exist unique intermediate states $w^{0}=u_{-}, w^{1}, \cdots, w^{n}=$ $u_{+}$and wave strength $\sigma^{k}$ such that

$(2.2)$

$w^{k}=\mathcal{R}^{k}\left(\sigma^{k}\right)\left(w^{k-1}\right)=w^{k-1}+\sigma^{k} r^{k}\left(w^{k-1}\right), r^{k}\left(w^{k-1}\right)=r^{k}\left(w^{k}\right), k=1,2, \cdots, n$, provided that $\Omega$ is a small neighborhood of $u_{-}$.

It is easy to verify that the solution $u(x, t)$ of the Riemann problem (1.1) and (2.1) is given by

$$
u(x, t)= \begin{cases}u_{-}, & \text {for } \frac{x}{t}<0 \\ \mathcal{R}\left(u_{-}, u_{+}\right)\left(\frac{x}{t}\right), & \text { for } \frac{x}{t} \in[0,1] \\ u_{+}, & \text {for } \frac{x}{t}>1 .\end{cases}
$$

Here

$$
\mathcal{R}\left(u_{-}, u_{+}\right)(\xi)=\mathcal{R}^{k}\left(z^{k}(\xi, 1)\right)\left(w^{k-1}\right) \text { for } \xi \in\left[\bar{\lambda}^{k-1}, \bar{\lambda}^{k}\right], \quad k=1,2, \cdots, n,
$$

and $z^{k}(x, t)$ is the unique (self similar) entropy solution to the scalar Riemann problem

$$
z_{t}+F^{k}(z)_{x}=0, \quad z(x, 0)= \begin{cases}0, & \text { for } x<0, \\ \sigma^{k}, & \text { for } x>0,\end{cases}
$$

with $F^{k}(\sigma)=\int_{0}^{\sigma} \lambda^{k}\left(\mathcal{R}^{k}(s)\left(w^{k-1}\right)\right) d s$.

In order to obtain a uniform BV estimate on the approximate solutions, we need to estimate the summation of wave strengths for all adjacent discontinuities at time $t=j \Delta t, j \in \mathbf{Z}_{+}$. This requires the relation between the wave strengths at time $t=(j+1) \Delta t$ and those at time $t=j \Delta t$. For the Godunov scheme, such a relation is easier to obtain because the definition of the Godunov scheme comes from the averaging of two Riemann solutions. For Lax-Friedrichs' scheme considered in this paper, we will first introduce an auxiliary state $\bar{u}_{i}^{j+1}$ similar to that of the Godunov scheme and then rewrite the expression (1.5) in terms of $\bar{u}_{i}^{j}$. Then, the relation is obtained by using the assumption (1.4) as explained in the following. 
Set

$$
\bar{u}_{i}^{j+1}=u_{i}^{j}-\left(f\left(u_{i}^{j}\right)-f\left(u_{i-1}^{j}\right)\right) .
$$

We have by integrating (1.1) over the rectangle

$$
\left[\left(i-\frac{1}{2}\right) \Delta x,\left(i+\frac{1}{2}\right) \Delta x\right] \times[j \Delta t,(j+1) \Delta t]
$$

that

$$
\bar{u}_{i}^{j+1}=\int_{0}^{1} \mathcal{R}\left(u_{i-1}^{j}, u_{i}^{j}\right)(\xi) d \xi .
$$

It is easy to see that if $u_{i-1}^{j}$ is connected to $u_{i}^{j}$ by a single $k-t h$ wave of strength $\sigma_{i-1, k}^{j}$, then

$$
\bar{u}_{i}^{j+1}=u_{i-1}^{j}+\left(1-\lambda^{k}\left(u_{i-1}^{j}, u_{i}^{j}\right)\right) \sigma_{i-1, k}^{j} r^{k}\left(u_{i-1}^{j}\right), \quad r^{k}\left(u_{i-1}^{j}\right)=r^{k}\left(u_{i}^{j}\right) .
$$

Here

$$
\lambda^{k}\left(u_{i-1}^{j}, u_{i}^{j}\right)=\int_{0}^{1} \lambda^{k}\left(u_{i-1}^{j}+s \sigma_{i-1, k}^{j} r^{k}\left(u_{i-1}^{j}\right)\right) d s .
$$

From (1.5) and (2.6), we can deduce that

$$
u_{i}^{j+1}=\frac{1}{2}\left(\bar{u}_{i}^{j+1}+\bar{u}_{i+1}^{j+1}\right)-\frac{1}{2}\left(u_{i}^{j}-u_{i-1}^{j}\right) .
$$

We now consider the variation between two grid points at time $(j+1) \Delta t$ by relating it to the variation of four grid points at the time $j \Delta t$. Given four consecutive states $u_{i-1}^{j}, u_{i}^{j}, u_{i+1}^{j}$ and $u_{i+2}^{j}$, let $u_{i}^{j+1}$ and $u_{i+1}^{j+1}$ be the two resulting states given by LaxFriedrichs' scheme (1.5). From (2.9), we have

$$
u_{i+1}^{j+1}=\frac{1}{2}\left(\bar{u}_{i+1}^{j+1}+\bar{u}_{i+2}^{j+1}\right)-\frac{1}{2}\left(u_{i+1}^{j}-u_{i}^{j}\right) .
$$

The strengths of the $k$-waves, $k=1,2, \cdots, n$, in the four Riemann problems $\left(u_{i-1}^{j}, u_{i}^{j}\right),\left(u_{i}^{j}, u_{i+1}^{j}\right),\left(u_{i+1}^{j}, u_{i+2}^{j}\right)$ and $\left(u_{i}^{j+1}, u_{i+1}^{j+1}\right)$ are denoted by $\sigma_{i-1, k}^{j}, \sigma_{i, k}^{j}$, $\sigma_{i+1, k}^{j}$ and $\sigma_{i, k}^{j+1}$, respectively. The intermediate states in the first three Riemann problems are denoted by $w_{i-1}^{j, 0}=u_{i-1}^{j}, w_{i-1}^{j, 1}, \cdots, w_{i-1}^{j, n-1}, w_{i-1}^{j, n}=u_{i}^{j}=w_{i}^{j, 0}, w_{i}^{j, 1}, \cdots$, $w_{i}^{j, n-1}, w_{i}^{j, n}=u_{i+1}^{j}=w_{i+1}^{j, 0}, w_{i+1}^{j, 1}, \cdots, w_{i+1}^{j, n-1}$, and $w_{i+1}^{j, n}=u_{i+2}^{j}$, respectively. Now we define the map which measures the change in the amount of waves in the $k-t h$ family produced by Lax-Friedrichs' scheme from $t=j \Delta t$ to $t=(j+1) \Delta t$ :

$$
\begin{aligned}
\Psi^{k}\left(\sigma_{i-1}^{j} ; \sigma_{i}^{j} ; \sigma_{i+1}^{j}\right)= & \sigma_{i, k}^{j+1}-\frac{1}{2}\left(1+\lambda^{k}\left(w_{i-1}^{j, k-1}, w_{i-1}^{j, k}\right)\right) \sigma_{i-1, k}^{j} \\
& -\frac{1}{2}\left(1-\lambda^{k}\left(w_{i+1}^{j, k-1}, w_{i+1}^{j, k}\right)\right) \sigma_{i+1, k}^{j},
\end{aligned}
$$

where $\sigma_{l}^{j}=\left(\sigma_{l, 1}^{j}, \cdots, \sigma_{l, n}^{j}\right), l=i-1, i, i+1$.

Under the assumption (1.4), we have the following main lemma in this paper.

Lemma 2.1. Under the assumption (1.4), there exist smooth functions $A_{p, q}^{k}, B_{p, q}^{k}$, $C_{p, q}^{k}, D_{p, q}^{k}, E_{p, q}^{k}$ and $F_{p, q}^{k}$, depending on $\sigma_{i-1}^{j}, \sigma_{i}^{j}$ and $\sigma_{i+1}^{j}$, such that

$$
\begin{aligned}
\Psi^{k}\left(\sigma_{i-1}^{j} ; \sigma_{i}^{j} ; \sigma_{i+1}^{j}\right)=\sum_{1 \leq p \neq q \leq n} & \left(A_{p, q}^{k} \sigma_{i-1, p}^{j} \sigma_{i-1, q}^{j}+B_{p, q}^{k} \sigma_{i-1, p}^{j} \sigma_{i, q}^{j}+C_{p, q}^{k} \sigma_{i-1, p}^{j} \sigma_{i+1, q}^{j}\right. \\
& \left.+D_{p, q}^{k} \sigma_{i, p}^{j} \sigma_{i, q}^{j}+E_{p, q}^{k} \sigma_{i, p}^{j} \sigma_{i+1, q}^{j}+F_{p, q}^{k} \sigma_{i+1, p}^{j} \sigma_{i+1, q}^{j}\right) .
\end{aligned}
$$


Proof. From the standard interpolation technique and a first order Taylor expansion (cf. [4), to prove (2.12) we only need to show that $\Psi^{k}\left(\sigma_{i-1}^{j} ; \sigma_{i}^{j} ; \sigma_{i+1}^{j}\right)$ satisfies $\Psi^{k}\left(0, \cdots, 0, \sigma_{i-1, k}^{j}, 0, \cdots, 0 ; 0, \cdots, 0, \sigma_{i, k}^{j}, 0, \cdots, 0 ; 0, \cdots, 0, \sigma_{i+1, k}^{j}, 0, \cdots, 0\right) \equiv 0$.

In fact, $\sigma_{s, l}^{j}=\sigma_{s, k}^{j} \delta_{k, l}(s=i-1, i, i+1)$ implies that

$$
\begin{cases}u_{i}^{j}=u_{i-1}^{j}+\sigma_{i-1, k}^{j} r^{k}\left(u_{i-1}^{j}\right), & r^{k}\left(u_{i-1}^{j}\right)=r^{k}\left(u_{i}^{j}\right), \\ u_{i+1}^{j}=u_{i}^{j}+\sigma_{i, k}^{j} r^{k}\left(u_{i}^{j}\right), & r^{k}\left(u_{i+1}^{j}\right)=r^{k}\left(u_{i}^{j}\right), \\ u_{i+2}^{j}=u_{i+1}^{j}+\sigma_{i+1, k}^{j} r^{k}\left(u_{i+1}^{j}\right), & r^{k}\left(u_{i+2}^{j}\right)=r^{k}\left(u_{i+1}^{j}\right)=r^{k}\left(u_{i}^{j}\right) .\end{cases}
$$

Furthermore, from (2.8) and (2.14) we deduce that

$$
\left\{\begin{array}{l}
\bar{u}_{i}^{j+1}=u_{i-1}^{j}+\left(1-\lambda^{k}\left(u_{i-1}^{j}, u_{i}^{j}\right)\right) \sigma_{i-1, k}^{j} r^{k}\left(u_{i-1}^{j}\right), \\
\bar{u}_{i+2}^{j+1}=u_{i+1}^{j}+\left(1-\lambda^{k}\left(u_{i+1}^{j}, u_{i+2}^{j}\right)\right) \sigma_{i+1, k}^{j} r^{k}\left(u_{i+1}^{j}\right) .
\end{array}\right.
$$

$$
\begin{aligned}
\begin{array}{c}
\text { Consequently, from }(2.9),(2.15), \text { and }(2.14) 1 \\
u_{i+1}^{j+1}-u_{i}^{j+1}=
\end{array}= & \frac{1}{2}\left\{\left(\bar{u}_{i+1}^{j+1}+\bar{u}_{i+2}^{j+1}\right)-\left(u_{i+1}^{j}-u_{i}^{j}\right)\right\}-\frac{1}{2}\left\{\left(\bar{u}_{i}^{j+1}+\bar{u}_{i+1}^{j+1}\right)-\left(u_{i}^{j}-u_{i-1}^{j}\right)\right\} \\
= & \frac{1}{2}\left(\bar{u}_{i+2}^{j+1}-\bar{u}_{i}^{j+1}\right)-\frac{1}{2}\left(u_{i+1}^{j}-2 u_{i}^{j}+u_{i-1}^{j}\right) \\
= & \frac{1}{2}\left\{u_{i+1}^{j}+\left(1-\lambda^{k}\left(u_{i+1}^{j}, u_{i+2}^{j}\right)\right) \sigma_{i+1, k}^{j} r^{k}\left(u_{i+1}^{j}\right)\right\} \\
& -\frac{1}{2}\left\{u_{i-1}^{j}+\left(1-\lambda^{k}\left(u_{i-1}^{j}, u_{i}^{j}\right)\right) \sigma_{i-1, k}^{j} r^{k}\left(u_{i-1}^{j}\right)\right\} \\
& -\frac{1}{2}\left(u_{i+1}^{j}-2 u_{i}^{j}+u_{i-1}^{j}\right) \\
= & \frac{1}{2}\left(1-\lambda^{k}\left(u_{i+1}^{j}, u_{i+2}^{j}\right)\right) \sigma_{i+1, k}^{j} r^{k}\left(u_{i+1}^{j}\right) \\
& -\frac{1}{2}\left(1-\lambda^{k}\left(u_{i-1}^{j}, u_{i}^{j}\right)\right) \sigma_{i-1, k}^{j} r^{k}\left(u_{i-1}^{j}\right)+\left(u_{i}^{j}-u_{i-1}^{j}\right) \\
= & \frac{1}{2}\left\{\left(1-\lambda^{k}\left(u_{i+1}^{j}, u_{i+2}^{j}\right)\right) \sigma_{i+1, k}^{j}+\left(1+\lambda^{k}\left(u_{i-1}^{j}, u_{i}^{j}\right)\right) \sigma_{i-1, k}^{j}\right\} r^{k}\left(u_{i-1}^{j}\right),
\end{aligned}
$$

which implies that the Riemann problem $\left(u_{i+1}^{j+1}, u_{i}^{j+1}\right)$ is solved by a single wave of family $k$ and with strength $\frac{1}{2}\left(1-\lambda^{k}\left(u_{i+1}^{j}, u_{i+2}^{j}\right)\right) \sigma_{i+1, k}^{j}+\frac{1}{2}\left(1+\lambda^{k}\left(u_{i-1}^{j}, u_{i}^{j}\right)\right) \sigma_{i-1, k}^{j}$. This shows that

$$
\sigma_{i, k}^{j+1}=\frac{1}{2}\left(1-\lambda^{k}\left(u_{i+1}^{j}, u_{i+2}^{j}\right)\right) \sigma_{i+1, k}^{j}+\frac{1}{2}\left(1+\lambda^{k}\left(u_{i-1}^{j}, u_{i}^{j}\right)\right) \sigma_{i-1, k}^{j} .
$$

This is (2.13) and completes the proof of Lemma 2.1.

From Lemma 2.1, we know that the strengths $\sigma_{i}^{j}(i \in \mathbf{Z}, \quad j \geq 1)$ satisfy the following systems of equations: For $k=1,2, \cdots, n$

$$
\sigma_{i, k}^{j+1}=\frac{1}{2}\left\{\left(1-\lambda^{k}\left(w_{i+1}^{j, k-1}, w_{i+1}^{j, k}\right)\right) \sigma_{i+1, k}^{j}+\left(1+\lambda^{k}\left(w_{i-1}^{j, k-1}, w_{i-1}^{j, k}\right)\right) \sigma_{i-1, k}^{j}\right\}+Q_{i, k}^{j} .
$$


Here the quadratic coupling terms are given by

$$
\begin{aligned}
Q_{i, k}^{j}=\sum_{1 \leq p \neq q \leq n} & \left(A_{p, q}^{k} \sigma_{i-1, p}^{j} \sigma_{i-1, q}^{j}+B_{p, q}^{k} \sigma_{i-1, p}^{j} \sigma_{i, q}^{j}+C_{p, q}^{k} \sigma_{i-1, p}^{j} \sigma_{i+1, q}^{j}\right. \\
& \left.+D_{p, q}^{k} \sigma_{i, p}^{j} \sigma_{i, q}^{j}+E_{p, q}^{k} \sigma_{i, p}^{j} \sigma_{i+1, q}^{j}+F_{p, q}^{k} \sigma_{i+1, p}^{j} \sigma_{i+1, q}^{j}\right)
\end{aligned}
$$

from which we can see that the increase in total variation induced by Lax-Friedrichs' scheme comes only from terms involving two different characteristic families.

To control the increase of total variation induced by $Q_{i, k}^{j}$, as in 4, the properties of solutions $\rho_{i, k}^{j}$ to the following linear homogeneous difference equation

$$
\rho_{i, k}^{j+1}=\frac{1}{2}\left(1+\lambda^{k}\left(w_{i-1}^{j, k-1}, w_{i-1}^{j, k}\right)\right) \rho_{i-1, k}^{j}+\frac{1}{2}\left(1-\lambda^{k}\left(w_{i+1}^{j, k-1}, w_{i+1}^{j, k}\right)\right) \rho_{i+1, k}^{j}
$$

plays an essential role.

It is easy to see that the solutions $\rho_{i, k}^{j}$ to the difference equation (2.18) satisfy

(i) Nonnegative Perserving: if $\rho_{l, k}^{j} \geq 0$ for any $l \in \mathbf{Z}, j \geq 0$, then $\rho_{i, k}^{j+1} \geq 0$ for any $i \in \mathbf{Z}$.

(ii) Conservation: $\sum_{i=-\infty}^{\infty} \rho_{i, k}^{j+1}=\sum_{i=-\infty}^{\infty} \rho_{i, k}^{j}$.

(iii) $\quad L^{1}$-contraction: $\sum_{i=-\infty}^{\infty}\left|\rho_{i, k}^{j+1}\right| \leq \sum_{i=-\infty}^{\infty}\left|\rho_{i, k}^{j}\right|$.

For the interaction between two different families, the following lemma is proved in [4]:

Lemma 2.2. Assume that $1 \leq p<q \leq n$ and that (1.3) holds. Let $\rho_{i, p}^{j}$ and $\rho_{i, q}^{j}$ denote the solutions of (2.18) with initial data $\rho_{i, p}^{0}$ and $\rho_{i, q}^{0}$, respectively. Then for fixed $\bar{m} \in \mathbf{Z}$, we have the estimate

$$
\sum_{m=0}^{j} \sum_{i=-\infty}^{\infty}\left|\rho_{i+\bar{m}, p}^{m}\right|\left|\rho_{i, q}^{m}\right| \leq C(p, q)\left(\sum_{i=-\infty}^{\infty}\left|\rho_{i, p}^{0}\right|\right)\left(\sum_{i=-\infty}^{\infty}\left|\rho_{i, q}^{0}\right|\right),
$$

where $C(p, q)$ satisfies

$$
C(p, q) \leq \max _{u, v \in \Omega}\left\{\frac{2}{\lambda^{q}(u)-\lambda^{p}(v)}\right\} .
$$

Based on Lemma 2.1 and Lemma 2.2, the total variation norm of the approximate solutions by Lax-Friedrichs' scheme can be estimated as the one for the Godunov scheme in [4]. For convenience of the reader, we give the estimations as follows; cf. [4].

We start by assuming that Lax-Friedrichs' scheme is well defined up to the time step $j$ and we let $C_{0}$ be a constant that dominates the absolute values of all $A_{p, q}^{k}$, $B_{p, q}^{k}, C_{p, q}^{k}, D_{p, q}^{k}, E_{p, q}^{k}$ and $F_{p, q}^{k}$.

Let

$$
V^{k}(j)=\sum_{i=-\infty}^{\infty}\left|\sigma_{i, k}^{j}\right|, \quad k=1,2, \cdots, n ;
$$

we have from (2.16) that

$$
V^{k}(j+1) \leq V^{k}(j)+\sum_{i=-\infty}^{\infty}\left|Q_{i, k}^{j}\right| \leq V^{k}(0)+\sum_{m=0}^{j} \sum_{i=-\infty}^{\infty}\left|Q_{i, k}^{m}\right| .
$$


Denote

$$
Q^{k}(j)=\sum_{m=0}^{j} \sum_{i=-\infty}^{\infty}\left|Q_{i, k}^{m}\right|
$$

we have from (2.17) that

$$
\begin{aligned}
Q^{k}(j) \leq & C_{0} \sum_{p \neq q} \sum_{m=0}^{j} \sum_{i=-\infty}^{\infty}\left(\left|\sigma_{i-1, p}^{m}\right|\left|\sigma_{i-1, q}^{m}\right|+\left|\sigma_{i-1, p}^{m}\right|\left|\sigma_{i, q}^{m}\right|+\left|\sigma_{i-1, p}^{m}\right|\left|\sigma_{i+1, q}^{m}\right|\right. \\
& \left.+\left|\sigma_{i, p}^{m}\right|\left|\sigma_{i, q}^{m}\right|+\left|\sigma_{i, p}^{m}\right|\left|\sigma_{i+1, q}^{m}\right|+\left|\sigma_{i+1, p}^{m}\right|\left|\sigma_{i+1, q}^{m}\right|\right) \\
\leq & 3 C_{0} \sum_{p \neq q} \sum_{\bar{m}=0}^{2} \sum_{m=0}^{j} \sum_{i=-\infty}^{\infty}\left|\sigma_{i+\bar{m}, p}^{m}\right|\left|\sigma_{i, q}^{m}\right| \\
& =: 3 C_{0} \sum_{p \neq q} \sum_{\bar{m}=0}^{2} E_{\bar{m}} .
\end{aligned}
$$

Now we turn to the estimate $E_{\bar{m}}$. For this, let $\Gamma^{k}\left(i, j ; i^{\prime}, j^{\prime}\right)$ be the corresponding Green kernel to (2.18), i.e., for $j \geq j^{\prime}, \Gamma^{k}\left(i, j ; i^{\prime}, j^{\prime}\right)=\rho_{i, k}^{j}$ is the solution of (2.18) at the node $(i, j)$, with initial data

$$
\rho_{i^{\prime}, k}^{j^{\prime}}=\delta_{i^{\prime}}^{j^{\prime}}(i)= \begin{cases}1, & \text { if } i=i^{\prime}, \\ 0, & \text { otherwise. }\end{cases}
$$

By Duhamel's principle we can write the solution of the linear inhomogeneous system (2.16) as

$$
\begin{aligned}
\sigma_{i, k}^{j} & =\sum_{l=-\infty}^{\infty} \Gamma^{k}(i, j ; l, 0) \sigma_{l, k}^{0}+\sum_{r=0}^{j-1} \sum_{l=-\infty}^{\infty} \Gamma^{k}(i, j ; l, r) Q_{l, k}^{r} \\
& =\alpha_{i, k}^{j}+\beta_{i, k}^{j} .
\end{aligned}
$$

Consequently

$$
\begin{aligned}
E_{\bar{m}} & \leq \sum_{m=0}^{j} \sum_{i=-\infty}^{\infty}\left(\left|\alpha_{i+\bar{m}, p}^{m}\right|\left|\beta_{i, q}^{m}\right|+\left|\alpha_{i+\bar{m}, p}^{m}\right|\left|\alpha_{i, q}^{m}\right|+\left|\beta_{i+\bar{m}, p}^{m}\right|\left|\alpha_{i, q}^{m}\right|+\left|\beta_{i+\bar{m}, p}^{m}\right|\left|\beta_{i, q}^{m}\right|\right) \\
& =: S_{\bar{m}}^{1}+S_{\bar{m}}^{2}+S_{\bar{m}}^{3}+S_{\bar{m}}^{4} .
\end{aligned}
$$

$S_{\bar{m}}^{l}(l=1,2,3,4)$ can be estimated by the following:

$$
\begin{aligned}
& S_{\bar{m}}^{4}=\sum_{m=0}^{j} \sum_{i=-\infty}^{\infty}\left|\sum_{r=0}^{m-1} \sum_{l=-\infty}^{\infty} \Gamma^{p}(i+\bar{m}, j ; l, r) Q_{l, p}^{r}\right|\left|\sum_{s=0}^{m-1} \sum_{h=-\infty}^{\infty} \Gamma^{q}(i, m ; h, s) Q_{h, p}^{s}\right| \\
\leq & \sum_{m=0}^{j} \sum_{i=-\infty}^{\infty}\left(\sum_{r=0}^{m-1} \sum_{l=-\infty}^{\infty} \Gamma^{p}(i+\bar{m}, j ; l, r)\left|Q_{l, p}^{r}\right|\right)\left(\sum_{s=0}^{m-1} \sum_{h=-\infty}^{\infty} \Gamma^{q}(i, m ; h, s)\left|Q_{h, q}^{s}\right|\right) \\
\leq & \sum_{r=0}^{j-1} \sum_{l=-\infty}^{\infty}\left|Q_{l, p}^{r}\right| \sum_{s=0}^{j-1} \sum_{h=-\infty}^{\infty}\left|Q_{h, q}^{s}\right|\left(\sum_{m=0}^{j} \sum_{i=-\infty}^{\infty} \Gamma^{p}(i+\bar{m}, m ; l, r) \Gamma^{q}(i, m ; h, s)\right) .
\end{aligned}
$$


We now have to estimate the term $\sum_{m=0}^{j} \sum_{i=-\infty}^{\infty} \Gamma^{p}(i+\bar{m}, m ; l, r) \Gamma^{q}(i, m ; h, s)$. For this, without loss of generality, we assume $s \geq r$ and in this case $\Gamma^{p}(i+\bar{m}, m ; l, r)$ is the solution of (2.18) (with $k=p$ ) at the time step $m$ and with data $\Gamma^{p}(i+\bar{m}, s ; l, r)$ at the time step $s, \Gamma^{q}(i, m ; h, s)$ is the solution of $(2.18)$ (with $k=q$ ) at the time step $m$ and with data $\delta_{h}^{s}(i)$ at the time step $s$. Thus from Lemma 2.2, we have

$$
\begin{aligned}
& \sum_{m=0}^{j} \sum_{i=-\infty}^{\infty} \Gamma^{p}(i+\bar{m}, m ; l, r) \Gamma^{q}(i, m ; h, s) \\
& \quad \leq C(p, q)\left(\sum_{i=-\infty}^{\infty} \Gamma^{p}(i+\bar{m}, s ; l, r)\right)\left(\sum_{i=-\infty}^{\infty} \delta_{h}^{s}(i)\right) \\
& \quad=C(p, q) \sum_{i=-\infty}^{\infty} \Gamma^{p}(i+\bar{m}, s ; l, r)=C(p, q) .
\end{aligned}
$$

Here we have used the conservation property of solutions to the linear homogeneous difference equation (2.18).

Substituting (2.28) into (2.27) yields

$$
S_{\bar{m}}^{4} \leq C(p, q)\left(\sum_{r=0}^{j-1} \sum_{l=-\infty}^{\infty}\left|Q_{l, p}^{r}\right|\right)\left(\sum_{s=0}^{j-1} \sum_{h=-\infty}^{\infty}\left|Q_{h, q}^{s}\right|\right) \leq C(p, q) Q^{p}(j-1) Q^{q}(j-1) .
$$

Similarly, by the $L^{1}$-contractive property of solutions to (2.18) and Lemma 2.2, we have

$$
\begin{gathered}
S_{\bar{m}}^{1} \leq C(p, q) V^{p}(0) V^{q}(0), \\
S_{\bar{m}}^{2} \leq C(p, q) V^{p}(0) Q^{q}(j-1),
\end{gathered}
$$

and

$$
S_{\bar{m}}^{3} \leq C(p, q) V^{q}(0) Q^{p}(j-1) .
$$

Putting (2.29)-(2.32) into (2.26), we have

$$
E_{\bar{m}} \leq C(p, q)\left(V^{p}(0)+Q^{p}(j-1)\right)\left(V^{q}(0)+Q^{q}(j-1)\right) .
$$

Thus

$$
\begin{aligned}
Q^{k}(j) & \leq 3 C_{0} \sum_{p \neq q} \sum_{\bar{m}=0}^{2} C(p, q)\left(V^{p}(0)+Q^{p}(j-1)\right)\left(V^{q}(0)+Q^{q}(j-1)\right) \\
& \leq C_{1} \sum_{p \neq q}\left(V^{p}(0)+Q^{p}(j-1)\right)\left(V^{q}(0)+Q^{q}(j-1)\right) .
\end{aligned}
$$

Here

$$
C_{1}=9 C_{0} \max _{1 \leq p \neq q \leq n, \quad u, v \in \Omega}\left\{\frac{2}{\left|\lambda^{q}(u)-\lambda^{p}(v)\right|}\right\} .
$$

Set

$$
V(j)=\sum_{k=1}^{n} V^{k}(j), \quad Q(j)=\sum_{k=1}^{n} Q^{k}(j)
$$


Then, we have from $(2.22),(2.34)$ and $(2.35)$ that

$$
\begin{gathered}
V(j) \leq V(0)+Q(j-1), \\
Q(j) \leq C_{2}(V(0)+Q(j-1))^{2},
\end{gathered}
$$

where $C_{2}=2 n C_{1}$.

It follows from (2.36) and (2.37) that, if the total amount of waves $V(0)$ in the initial data is sufficiently small, then $Q(j)$, and hence also the total amount of waves $V(j)$ remains small for all time steps. Thus (1.7) holds for all $j \in \mathbf{Z}^{+}$and $u_{i}^{j}$ remains in $\Omega$ for all $i \in \mathbf{Z}, j \geq 0$ which means that the scheme is well defined for all $i \in \mathbf{Z}, j \in \mathbf{Z}^{+}$. Based on these uniform BV estimates, the $L^{1}$-stability result (1.8) follows from the previous works; cf. [3, 4, 5, 6, 11]. This completes the proof of Theorem 1.1.

\section{REFERENCES}

1. S. Bianchini and A. Bressan, A center manifold technique for tracing viscous waves, preprint 2001.

2. S. Bianchini and A. Bressan, BV solutions for a class of viscous hyperbolic systems, Indiana Univ. Math. J. 49 (4) (2000), 1673-1713. MR 2002c:35175

3. A. Bressan, Hyperbolic Systems of Conservation Laws. The One Dimensional Cauchy Problem, Oxford University Press, 2000. MR 2002d:35002

4. A. Bressan and H. K. Jenssen, On the convergence of Godunov scheme for nonlinear hyperbolic systems, Chin. Ann. Math. 21B (3) (2000), 269-284. CMP 2001:02

5. A. Bressan and P. LeFloch, Uniqueness of weak solutions to systems of conservation laws, Arch. Rational Mech. Anal. 140 (1997), 301-317. MR 98m:35125

6. A. Bressan, T.-P. Liu and T. Yang, $L^{1}$ stability estimates for $n \times n$ conservation laws, Arch. Rational Mech. Anal. 149 (1999), 1-22. MR 2000g:35139

7. R. J. DiPerna, Convergence of approximate solutions to conservation laws, Arch. Rational Mech. Anal. 82 (1983), 27-70. MR 84k:35091

8. J. Glimm, Solutions in the large for nonlinear hyperbolic systems of equations, Comm. Pure Appl. Math. 18 (1965), 697-715. MR 33:2976

9. P. D. Lax, Hyperbolic systems of conservation laws II, Comm. Pure Appl. Math. 10 (1957), 537-566. MR 20:176

10. L.-W. Lin and T. Yang, Convergence of the Lax-Friedrichs' scheme for isentropic gas dynamics in Lagrangian coordinates, Commun. Partial Differential Equations 16 (8 \& 9) (1991), 1441-1460. MR 92k:35181

11. T.-P. Liu and T. Yang, Well-posedness theory for hyperbolic conservation laws, Comm. Pure Appl. Math. 52 (12) (1999), 1553-1586. MR 2000m:35126

12. O. A. Oleinik, Discontinuous solutions of nonlinear differential equations, Amer. Math. Soc. Transl. Ser. 2, 26 (1963), 95-172. MR 27:1721

13. D. Serre, Systems of Conservation Laws, I, II, Cambridge University Press, Cambridge, 2000. MR 2000g:35142 MR 2001c:35146

14. B. Temple, Systems of conservation laws with invariant submanifolds, Trans. Amer. Math. Soc. 280 (1983), 781-795. MR 84m:35080

Department of Mathematics, City University of Hong Kong, Hong Kong

E-mail address: matyang@math.cityu.edu.hk

Wuhan Institute of Physics and Mathematics, The Chinese Academy of Sciences, Wuhan, People's Republic of China

E-mail address: hjzhao@wipm.ac.cn

Laboratory of Nonlinear Analysis and Department of Mathematics, Central China Normal University, Wuhan, People's Republic of China

E-mail address: cjzhu@ccnu.edu.cn 